Ann. Génét. Sél. anim., I975, 7 (2), I67-180.

\title{
ÉTUDE DES MARQUEURS GÉNÉTIQUES SANGUINS DANS DEUX RAGES DE PONEYS DE POLOGNE
}

\author{
Luba PODLIACHOUK, Marie KAMINSKI* et J. ZWOLINSKI** \\ avec la collaboration technique de $M^{\text {mes }} \mathrm{R}$. BeAud, F. Pigache, \\ Mile M. Sykiotis, MM. J. M. Genna et G. Cholewinski \\ Centre de Biologie et Pathologie infectieuse des Équidés, \\ Institut Pasteur, Paris (1) \\ * Laboratoire d'Enzymologie, C. N. R. S., \\ 91190 Gif sur Yvette (France) \\ ** Akademia Rolnicza, Poznan (Pologne)
}

\section{RÉSUMÉ}

L'étude des marqueurs génétiques sanguins, comprenant 24 antigènes érythrocytaires répartis en 7 systèmes génétiques et 7 non classés, de 4 systèmes de protéines et enzymes polymorphes du sérum et de l'hémolysat, a été effectuée sur 63 chevaux de race Fjording et $8 \mathrm{r}$ chevaux de race $H u c u l$, les deux groupes provenant des élevages polonais.

On a démontré des différences marquées entre les deux races dans les fréquences des facteurs, des phénotypes ou des allèles, dans plusieurs systèmes génétiques.

La comparaison avec d'autres races de chevaux a permis de constater des similitudes avez des chevaux de trait plutôt qu'avec des chevaux " de sang ".

\section{INTRODUCTION}

Des études des marqueurs génétiques chez des chevaux de races diverses ont été publiées depuis I957; il s'agissait surtout des antigènes érythrocytaires. Les techniques électrophorétiques et histochimiques de détermination des systèmes de protéines et enzymes polymorphes du sérum et de l'hémolysat ont été mises au point à partir de 1964 .

En 1972 a été entreprise, sous l'égide de la Société d'Encouragement pour l'Amélioration des races de chevaux en France, une étude comparative, à l'aide du plus grand

(1) Adresse actuelle : Laboratoire de Génétique biochimique, I. N. R. A., C. N. R. Z., 78350 Jouy en Josas (France). 
nombre de marqueurs génétiques disponibles, portant sur un large éventail de races : depuis les chevaux de " sang chaud " ou races légères, aux chevaux à "sang froid " ou races lourdes, dits autrefois chevaux de trait (PoDliachouk et KAMINSKI, I972 ; KAMINSKI et al., r976).

Pour les races les plus importantes, tels le Pur Sang Anglais, 1'Arabe, ou le Trotteur - tous chevaux de courses - des populations originaires des pays divers furent également comparées (Podilachouk et al., I974 ; Podirachouk et al., I976).

L'intérêt de telles études est de fournir des données sur l'évolution de l'espèce, résultant de plusieurs causes, tels que le milieu, la sélection imposée par l'homme, la dérive, etc.

On peut, en effet, à l'aide des renseignements sur la répartition des facteurs, phénotypes ou allèles, déterminer les relations phylogénétiques entre diverses races.

A côté des races les plus répandues et connues, il semblait important d'analyser dans cette optique les divers représentants des Poneys. Ces chevaux ont un double intérêt, historique et actuel : ils dérivent pour la plupart des chevaux locaux primitifs et sont, dans beaucoup de pays, objet d'élevage intensif en vue de sport, des jeux, etc.

Deux races de Poneys ont été étudiées ; le Fjording, a déjà été analysé antérieurement (Podifachouk et al., I962; BRAEND, I964), cependant le nombre total de marqueurs génétiques utilisé dans le travail présent est de beaucoup supérieur.

\section{MATÉRIEL}

Les deux races proviennent des élevages polonais. Leurs berceaux d'origine sont très distants géographiquement (1), mais les deux races se sont fixées dans des conditions d'environnement similaires : région montagneuse, climat rude.

\section{I. - Race Fjording ou Poney norvégien}

Chevaux d'origine nordique, croisés aux cours des âges avec beaucoup d'autres races notam. ment des Ardennais, mais aussi des PSA. Malgré ces apports le Fjording a conservé un type morphologique archaïque et surtout remarquablement homogène. Le manque de variantes dans la robe est considéré comme caractéristique des chevaux primitifs. Du point de vue de l'élevage, on considère que les meilleurs produits sont obtenus en croisant les Fjordings avec les chevaux de "sang froid", surtout Ardennais. Actuellement les Fjordings sont élevés en Norvège en tant que race pure.

L'élevage polonais date d'après la seconde guerre mondiale.

Nous avons examiné 63 échantillons de sang. Parmi eux il y avait 2 séries complètes de familles l'une d'un étalon de race ardennaise, avec 13 juments et 16 produits, l'autre avec 5 juments et 5 produits.

\section{II. - Race Hucul ou Poney des Carpathes}

Ces chevaux font partie d'un groupe qualifié de "tarpanidés ». Le type de petit cheval montagnard est connu depuis le Xvis ${ }^{\mathbf{e}}$ siècle dans une région habitée par les Huculs, dans les Carpathes. Plusieurs souches de chevaux divers : prises de guerre, restes des armées d'envahissement, etc. ont formé cette race, qui s'est fixée sous l'influence prépondérante des conditions d'environne-

(1) Les précisions sur l'origine et les caractères de ces deux races sont tirées de:

- W. Pruski, Hodowla Koni, Panstwowe Wydawnictwo Rolnicze i Lesne, Warszawa, 1960.

- J. Zwolinski, Hodowla Koni, Panstwowe Wydawnictwo Rolnicze i Lesne, Warszawa, 1971. 
ment particulières dans une région au climat rude. Le type résultant est un cheval d'aspect également plutôt primitif. Il est élevé en Roumanie, Tchécoslovaquie, U. R. S. S. et Pologne. L'élevage polonais systématique date du $\mathrm{xIx}^{\mathrm{e}}$ siècle; le livre généalogique compte environ 600 animaux actuellement.

Nous avons examiné 8 I échantillons de sang; parmi eux il y avait de nombreuses familles, dont 2 séries complètes, l'une avec I I juments et 19 produits, l'autre avec 6 juments et Io produits.

\section{MÉTHODES}

\section{I. - Antigènes érythrocytaires}

La méthodologie et la liste des facteurs sont rapportés ailleurs (Podliachouk et al., I974; PoduAachouk et al., 1976). Remarquons que dans le présent travail nous nous conformons aux décisions prises en juin I974 lors de la XIVe Conférence de l'ISABR (International Society for Animal Blood Group Research) où des nouvelles règles de nomenclature pour I 5 facteurs ont été adoptées. Dans le tableau I figurent l'ancienne et la nouvelle nomenclatures.

\section{II. - Protéines et enzymes polymorphes du sérum et de l'hémolysat}

Les méthodes ont été rapportées précédemment (Kaminski et al., 1974). Parmi les systèmes sériques, l'estérase avait été déterminée sur tous les échantillons à pH 8,5 et 4,5; parmi les enzymes de l'hémolysat, la phosphohexose isomérase seule a été déterminée sur la totalité des échantillons.

\section{RÉSULTATS}

\section{A. - Groupes sanguins}

Dans le présent travail 24 facteurs (antigènes érythrocytaires) ont été déterminés : $I_{4}$ sont désignés suivant une nomenclature internationale et ro correspondent à des réactifs personnels (L. PodLIAChouK). Ces facteurs sont répartis en 7 systèmes génétiques. De plus, 7 autres réactifs personnels ont permis de détecter des facteurs non encore classés dans les systèmes connus et décrits (tabl. r).

Dans le système $\mathrm{A}, \mathrm{P}$ et $\mathrm{Q}$, nous avons rencontré des globules (échantillons d'érythrocytes) ne présentant aucun antigène détectable. Ces systèmes sont vraisemblablement ouverts (LARSEN, I97I).

L,e système $\mathrm{D}$, contenant io facteurs, est un système fermé (SANDBERG, 1973).

Les fréquences de divers facteurs sont présentées dans le tableau I ; un résumé des différences principales entre les deux races basées sur les fréquences et la répartition des facteurs sont présentés dans le tableau 2.

On constate que les facteurs $\mathrm{G}$ et Df manquent chez les Fjordings et chez les Huculs. Les facteurs Qa, 12 et 20 , absents chez les Fjordings, sont assez fréquents chez les Huculs. Par contre, les facteurs I7, $\mathrm{Db}, \mathrm{Ka}, \mathrm{Pb}, 2 \mathrm{I}$ et 23, absents ou rares chez les Huculs, sont présents chez les Fjordings. Enfin les facteurs I, Aa, Dc, J, $\mathrm{De}$, Io et Ua sont fréquents chez les Fjordings et les Huculs, mais la différence de fréquence est du simple au double d'une race à l'autre.

Pour les Fjordings nos résultats se rapprochent de ceux de l'étude précédente (Podliachouk et al., 1962), où cependant le nombre total des facteurs était assez limité. 
I. PODLIACHOUK, M. KAMINSKI, J. ZWOLINSKI

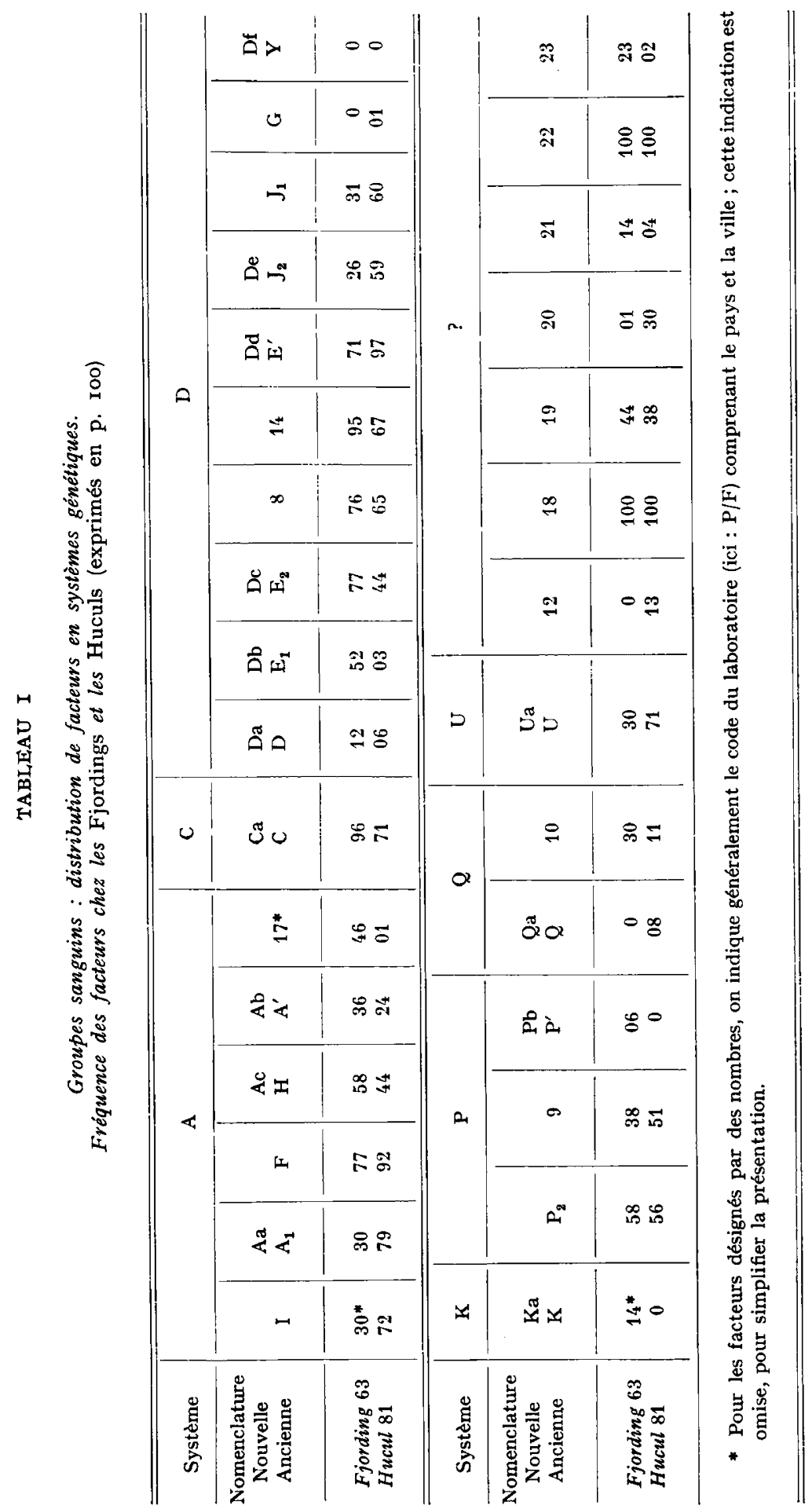


Par la présence des facteurs Ac et $\mathrm{Da}$ et absence de G, Df et Qa les Fjordings se rapprochent des chevaux de trait; cependant, ils possèdent aussi le facteur $\mathrm{Db}$, fréquent surtout chez les chevaux de sang. Quant aux Huculs, ils possèdent Da et Ac et manquent, totalement ou presque, de facteurs $\mathrm{Db}, \mathrm{G}, \mathrm{Df}$ et $\mathrm{Qa}$ : ainsi ils se rapprochent beaucoup des chevaux de trait (PoDLIACHouk et KAMINSKI, I972).

TABLEAU 2

Principales différences

dans les fréquences des facteurs de groupes sanguins entre les deux races

\begin{tabular}{l|r|r|r|r|r|r|r|r|r}
\hline \hline & $\mathrm{Qa}$ & 12 & 20 & 17 & $\mathrm{Db}$ & $\mathrm{Ka}$ & $\mathrm{Pb}$ & 21 & 23 \\
\hline & & & & & & & & \\
\hline $\begin{array}{l}\text { Fjording } \\
\text { Hucul }\end{array}$ & 08 & 13 & 30 & 01 & 03 & 0 & 0 & 04 & 02 \\
\hline
\end{tabular}

(1) Fréquences exprimées en p. 100.

L'étude de la transmission héréditaire des facteurs de groupes sanguins dans les familles a permis de démontrer que certains facteurs sont hérités en groupes et constituent ainsi des phénogroupes contrôlés par des allèles. Pour simplifier le texte, nous désignons les allèles de la même manière que les phénogroupes.

TABLEAU 3

Présence des allèles des systèmes de groupes sanguins $A, P$ et $Q$ dans les deux races

\begin{tabular}{|c|c|c|c|c|c|c|}
\hline & \multicolumn{6}{|c|}{ Système A } \\
\hline & I Aa $F$ & Aa $F$ & $\mathrm{Ab} 17$ & $A b$ & 17 & F Ac \\
\hline \multirow[t]{3}{*}{$\begin{array}{l}\text { Fjording } \\
\text { Hucul }\end{array}$} & $\stackrel{+}{++}$ & $\overline{+}$ & $\begin{array}{c}+++ \\
\pm\end{array}$ & $\overline{t+}+$ & + & $\begin{array}{l}+++ \\
+++\end{array}$ \\
\hline & \multicolumn{3}{|c|}{ Système P } & \multicolumn{3}{|c|}{ Système Q } \\
\hline & $\mathrm{P}_{2} 9$ & $P_{2}$ & $\mathrm{P}^{\prime}$ & Qa 10 & Qa & 10 \\
\hline $\begin{array}{l}\text { Fjording } \\
\text { Hucul }\end{array}$ & $\begin{array}{l}+++ \\
+++\end{array}$ & $\begin{array}{l}+++ \\
+++\end{array}$ & \pm & - & $\overline{+}$ & $\begin{array}{c}+++ \\
+\end{array}$ \\
\hline
\end{tabular}

Certains systèmes sont bi-alléliques $(\mathrm{C}, \mathrm{K}, \mathrm{U})$, d'autres multi-allèliques. Le premier allèle décrit fut AF (PoDlıachouk, I957); ensuite une série d'allèles décrite par SToRmont et Suzuki (I964) fut classée en systèmes A, P et Q. Récemment, le 
système D a été analysé par SANDBERG (1973-1974); cet auteur a mis en évidence dans ce système 6 facteurs formant 8 allèles (plus deux rares non décrits). Dans notre laboratoire, le système $\mathrm{D}$ comprend ro facteurs et 9 allèles (PoDliachouk et al., I974).

Les tableaux 3 et 4 présentent la liste des allèles connus actuellement et leur répartition dans les deux races étudiées.

TABLEAU 4

Fréquence des allèles du système $D$ dans les deux races

\begin{tabular}{l|c|c}
\hline \multicolumn{1}{c|}{ Allèles } & $\begin{array}{c}\text { Fjording } \\
\mathrm{N}=55\end{array}$ & $\begin{array}{c}\text { Hucul } \\
\mathrm{N}=54\end{array}$ \\
\hline Dd & - & 0,222 \\
Da Dd & 0,064 & 0,028 \\
Dd De & - & - \\
Dd Df & -300 & 0,028 \\
Db Dc 14 & 0,236 & 0,171 \\
Dc 8 14 & 0,264 & 0,166 \\
$814 \mathrm{Dd}$ & 0,136 & 0,376 \\
Dd J De & - & 0,009 \\
Dc 8 $14 \mathrm{De} \mathrm{G}$ & & \\
\hline
\end{tabular}

La différence la plus marquée entre les deux races est la présence de l'allèle $\mathrm{Ab}$ chez les Huculs uniquement alors que l'allèle I7 n'existe que chez les Fjordings.

Le système $P$ ne permet de montrer aucune différence particulière entre les deux races. Dans le système $Q$ les Fjordings ne possèdent pas l'allèle $Q a$.

En ce qui concerne le système $D$, on constate d'abord que sur les 9 allèles que nous avons trouvé chez les familles de chevaux Pur Sang Anglais, trotteurs et Boulonnais (Podriachouk et al., I974; Podliachouk et al., 1976) on ne trouve que 5 chez les Fjordings et 7 chez les Huculs.

Les allèles les plus fréquents chez les Fjordings sont Db Dc I4 ; 8 I4 Dd ; Dc 8 I4 ; chez les Huculs ce sont $\mathrm{Dd} \mathrm{J}_{1}$ De et $\mathrm{Dd}$, ce dernier complètement absent chez les Fjordings. (Le calcul des fréquences a été fait sur le matériel familial).

Le tableau 5 rassemble quelques exemples de détermination de génotypes, soit directement à partir des phénotypes : cas a et c, soit par analyse des familles : cas b et $d$.

Dans le cas $b, l^{\prime}$ un des deux génotypes de l'étalon, théoriquement possibles : De $8 \mathrm{I} 4 / \mathrm{Dd}$, est exclu par le génotype du produit, ne comprenant aucun de ces deux allèles, bien que l'on ne puisse définir avec certitude le génotype de la jument.

Dans le cas $d$, le produit ne possédant qu'un seul facteur : Dd, son génotype est forcément à l'état homozygote, donc $\mathrm{Dd} / \mathrm{Dd}$; on peut ainsi exclure les génotypes paternel et maternel ne contenant pas cet allèle. 
TABLEAU 5

Système $D$ de groupes sanguins : détermination de génotype dans les familles

\begin{tabular}{|c|c|c|c|c|}
\hline Race & Nom & No & Phénotype & Génotype \\
\hline a) Fjording & Lyngfax & 16 & $\mathrm{Da} \mathrm{Db} \mathrm{Dc} 14 \mathrm{Dd}$ & $\mathrm{Da} \mathrm{Dd} / \mathrm{Db} \mathrm{Dc} 14$ \\
\hline b) Arden. $\hat{\sigma}$ & Blum & 15 & Dc 814 Dd & $\begin{array}{l}\text { De } 814 / \mathrm{Dd} \\
\text { ou } \\
\text { De } 814 / 8 \quad 14 \quad \mathrm{Dd}\end{array}$ \\
\hline $\begin{array}{l}\text { Hucul }+ \\
\text { Hucul Pr. }\end{array}$ & $\begin{array}{l}\text { Wilbra } \\
\text { Wala }\end{array}$ & 36 & $\begin{array}{l}\text { Dd } J_{1} \text { De } \\
814 \text { Dd } J_{1} \text { De }\end{array}$ & $\begin{array}{l}\text { Dd } J_{1} \mathrm{De} / \mathrm{Dd} \\
\quad \text { ou } \\
\begin{array}{lll}\mathrm{Dd} & \mathrm{J}_{1} \mathrm{De} / \mathrm{Dd} J_{1} \mathrm{De} \\
8 & 14 \mathrm{Dd} / \mathrm{Dd} \mathrm{J}_{1} \mathrm{De}\end{array}\end{array}$ \\
\hline c) $\mathrm{Hucul}$ & Goral & 179 & $814 \mathrm{Dd} \mathrm{J}_{1} \mathrm{De}$ & $814 \mathrm{Dd} / \mathrm{Dd} \mathrm{J}_{1} \mathrm{De}$ \\
\hline d) $H u c u l$ oे & Hroby V22 & 159 & $814 \mathrm{Dd}$ & $\begin{array}{l}814 \mathrm{Dd} / \mathrm{Dd} \\
\text { ou } \\
814 \mathrm{Dd} / 814 \mathrm{Dd}\end{array}$ \\
\hline Hucul 우 & Maciejowa & 153 & $\mathrm{Dd} \mathrm{J}_{\mathrm{x}} \mathrm{De}$ & 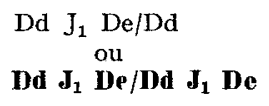 \\
\hline Hucul Pr. & Fregata & 105 & $\mathrm{Dd}$ & $\mathrm{Dd} / \mathrm{Dd}$ \\
\hline
\end{tabular}

Caractère gras : génotype exclu.

B. - Systèmes polymorphes du sérum

Le tableau 6 présente les fréquences des allèles et la répartition des phénotypes chez les deux races étudiées, pour les systèmes d'albumine, de transferrine et d'estérase. Pour l'albumine seules les bandes $\mathrm{F}$ et $\mathrm{S}$ ont été observées ; pour la transferrine la bande $\mathrm{M}$ n'a pas été rencontrée ; pour l'estérase, à $\mathrm{pH} 8,5$ les bandes $\mathrm{F}$, I et $\mathrm{S}$ ont été observées et 1'allèle silencieux récessif $\mathrm{Fs}_{\mathrm{s}}{ }^{\circ}$ a été supposé chez une jument et son produit ; à pH 4,5 les bandes F, G et I ont été observées.

\section{Albumine.}

Les fréquences allèliques sont pratiquement identiques pour les deux races; en ce qui concerne la répartition des phénotypes, le calcul de $\chi^{2}$ a démontré l'excès des hétérozygotes chez les Huculs $\left(\chi^{2}=7,2, n=\mathrm{I}\right.$, probabilité $\left.0,0 \mathrm{I}-0,00 \mathrm{I}\right)$.

\section{Transferrine.}

On remarque que le nombre total de phénotypes est bien moins élevé chez les Fjordings que chez les Huculs, 6 contre ro.

Dans les deux races c'est l'allèle Tf ${ }^{\mathrm{F}}$ qui est nettement prédominant; c'est d'ailleurs l'allèle le plus fréquent dans la plupart des races étudiées (BRAEND, I973). 
TABLEAU 6

Répartition de phénotypes et fréquences allèliques pour les systèmes polymorphes du sérum dans les deux races

Les effectifs sont de 63 pour les Fjordings, 8I pour les Huculs

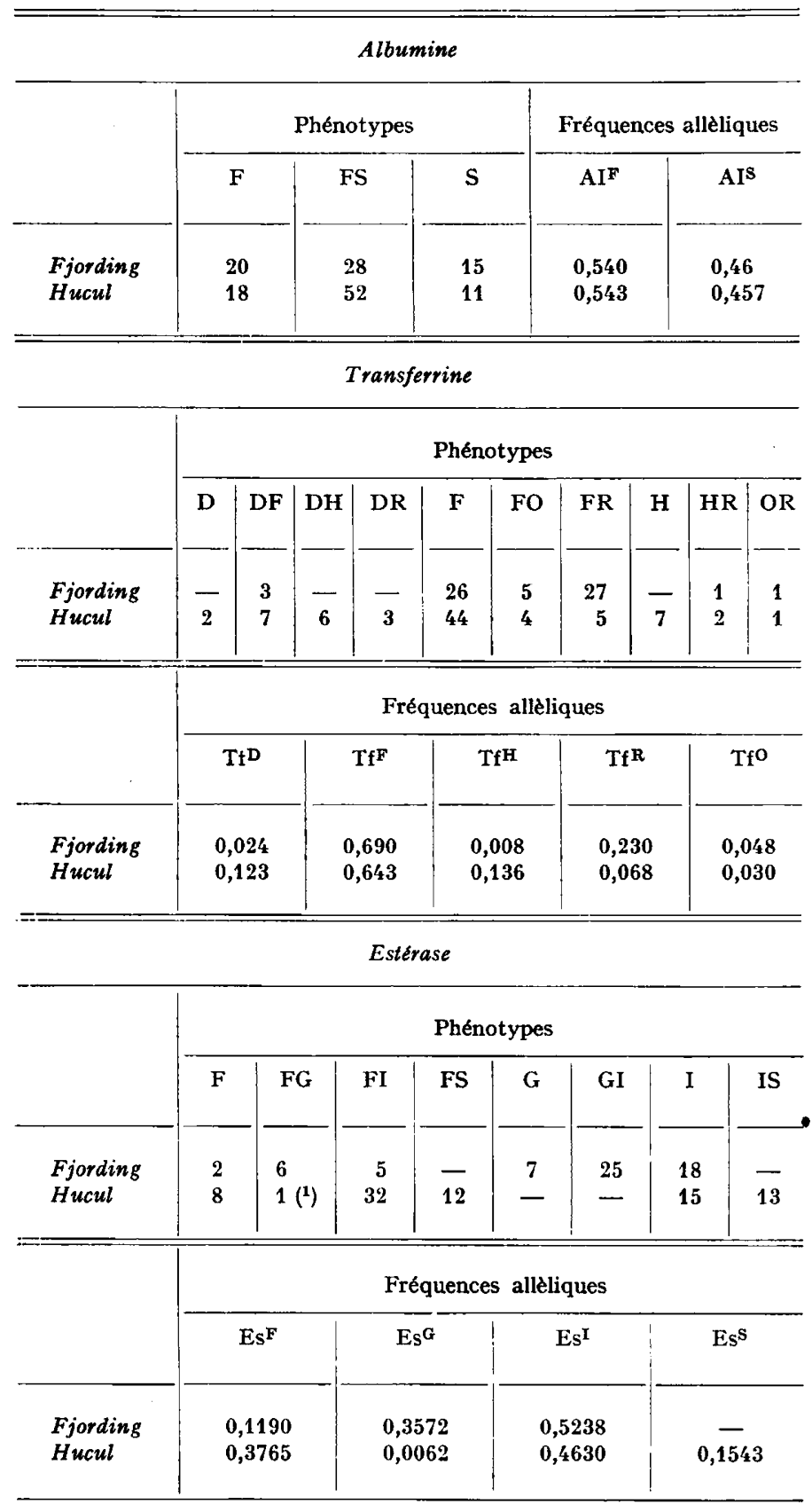

(1) Produit de l'étalon FS et de mère inconnue. 
Les fréquences de $\mathrm{Tf}^{\mathrm{F}}$ chez les Fjordings et les Huculs sont très voisines, cependant la fréquence des homozygotes $\mathrm{FF}$ est inférieure chez les Fjordings (39 p. roo) à celle chez les Huculs (54 p. roo).

Nos résultats sur les Fjordings peuvent être rapprochés de ceux de BRAEND (r964) sur les Fjordings norvégiens : les deux populations montrent une fréquence relativement élevée de $\operatorname{Tf}^{R}$ ( 23 p. Ioo et I9 p. roo). Cet allèle est rare chez les chevaux de sang et fréquent chez les chevaux de trait. L'autre bande lente, $O$, est également rare chez les Fjordings norvégiens et les polonais (Tfo $=4$ p. Ioo). Cependant les résultats diffèrent sensiblement pour $\operatorname{Tf}^{D}$ dont la fréquence est $2 \mathrm{p}$. Ioo chez les polonais et I $_{5} \mathrm{p}$. Ioo chez les norvégiens.

La proportion d'hétérozygotes est de $34 \mathrm{p}$. Ioo pour les $H$ uculs et près de $60 \mathrm{p}$. Ioo pour les Fjordings. Chez les Fjordings norvégiens, cette proportion est de même ordre : 54 p. Ioo, ceci pour 4 allèles seulement.

Dans l'ensemble, la répartition des fréquences des 5 allèles de transferrine chez les Fjordings polonais se rapproche le plus de celle des Fjordings norvégiens (BRAEND, r964), et diffère de résultats obtenus chez d'autres races de Poneys, tels les chevaux Islandais (HESSELHOLT, I967), les poneys Basuto (Osterhoff et al., I970), les Poneys de Gotland (BEnGTsson et al., I968), et les chevaux de montagne de Bulgarie (DoBREv et al., I970), ainsi que des Poneys Welsh et Shetland des Pays Bas (Burs, I973). Cette répartition diffère également nettement de celle observée chez les Tarpans (TomasZEWSKA-GUSZKIEWICZ et al., I970), race simili-archaïque de petits chevaux, créée et élevée en Pologne. Il faut cependant remarquer qu'à l'exception des Tarpans, toutes ces races présentent des fréquences voisines de $\operatorname{Tf}^{R}$ : entre $I_{5}$ et $20 \mathrm{p}$. IOo.

En ce qui concerne les Huculs, le " profil " allèlique de transferrine semble particulier à cette race et diffère des races étudiées (BRAEND, I973) par la fréquence de $\operatorname{Tf}^{F}$ dépassant $60 \mathrm{p}$. Ioo et, simultanément, les fréquences égales de $\operatorname{Tf}^{\mathrm{D}}$ et $\operatorname{Tf}^{\mathbf{H}}$ : I2-I3 p. roo.

\section{Estérase.}

La répartition des allèles d'estérase diffère foncièrement chez les deux races : on trouve chez les Fjordings un peu de Es ${ }^{F}$, beaucoup de $\mathrm{E}_{S^{\mathrm{G}}}$ et pas de Es $\mathbf{s}^{\mathrm{s}}$, alors que chez les Huculs il y a une assez forte fréquence de $\mathrm{Es}^{\mathrm{s}}$, beaucoup de Es $\mathrm{s}^{\mathrm{F}}$ et pas de $E_{S^{G}}$ sauf chez un seul animal dont la mère n'a pas été étudiée. Les fréquences de Es ${ }^{I}$, l'allèle le plus répandu d'estérase, sont d'environ 50 p. roo dans chaque race, ce qui les rapproche davantage des chevaux de trait que de sang (Podilachouk et KAMINSKI, I972 ; PoDliachouK et al., I974; KAMINSKI, I975).

La fréquence de $35 \mathrm{p}$. Ioo pour $\mathrm{Es}_{\mathrm{S}^{\mathrm{G}}}$ chez les Fjordings paraît remarquablement forte : parmi les II races que nous avons étudiées (KAMINSKI, I975), seuls les Percherons ont donné $34 \mathrm{p}$. Ioo ; les autres races de chevaux de trait avaient $27 \mathrm{p}$. I0o (Ardennais), 24 p. Ioo (Bretons), 25 p. Ioo (Boulonnais). Les chevaux de demi-sang (Trotteurs, Selle Français) en avaient environ Io p. I00; BRAEND (I964) a rapporté un taux analogue pour les Trotteurs norvégiens : I2 p. Ioo. Chez les chevaux de sang (Pur Sang Anglais) ce taux tombe à o (Podliachouk et Kaminski, r972 ; PodliacHouk et al., 1974).

La fréquence de $\mathrm{ES}^{\mathrm{G}}$ ne peut être directement comparée aux données rapportées dans la littérature, car on y trouve généralement la fréquence de $\mathrm{Es}_{\mathbf{S}}{ }^{\mathrm{F}}$ obtenue d'après l'analyse à $\mathrm{pH}$ alcalin. Dans ces conditions les bandes $\mathrm{F}$ et $\mathrm{G}$ sont confond ues et le 
$E s_{a l c}^{F}$. correspond à la fréquence globale $\mathrm{Es}_{\mathrm{ac}}^{\mathrm{F}}$. $+\mathrm{Es}_{\mathrm{ac}}^{\mathrm{G}}$. (alc. $=\mathrm{pH} \mathrm{8,5}$ et ac. $\left.=\mathrm{pH} 4,5\right)$.

En effectuant une telle addition pour les Fjordings, on trouve $\mathrm{Es}_{\mathrm{s}}^{\mathrm{F}}+\mathrm{Es}^{\mathrm{G}}=$ 46 p. roo. Cette valeur n'est dépassée ou atteinte que par les chevaux de trait. Ainsi on trouve : 47,5 p. roo chez les Ardennais, $3^{8}$ p. Ioo chez les chevaux "North Swedish" et 23 p. Ioo chez les Poneys Gotland (Bengtsson et al., r968) ; 45 p. Ioo chez les Percherons d'Afrique du Sud (OSTERhoff et al., I967) et 28 p. Ioo pour les Poneys Shetland (Buts, I973).

Nos résultats personnels confirment la fréquence élevée des bandes rapides d'estérase chez les chevaux de trait $=50 \mathrm{p}$. Ioo $\left(\mathrm{ES}_{\mathrm{S}^{\mathrm{F}}}+\mathrm{E}_{\mathrm{S}^{\mathrm{G}}}\right)$ chez les Ardennais, 54 p. Ioo chez les Bretons, 47 p. Ioo chez les Percherons et 36 p. Ioo chez les Cobs.

Les Fjordings montrent une proportion de 57 p. Ioo d'hétérozygotes pour l'estérase, en tenant compte de résultats obtenus à $\mathrm{pH}_{4,5}$; ce taux est de $50 \mathrm{p}$. IoO si l'analyse est faite à $\mathrm{pH} 8,5$.

La transmission de l'estérase chez les Fjordings a été étudiée dans I $_{5}$ familles (2 étalons) (tabl. 7). Dans un cas de croisement d'un étalon de phénotype FI avec

TABLEAU 7

Transmission d'allèles d'estérase dans les familles de Fjordings et Huculs

\begin{tabular}{|c|c|c|c|}
\hline \multicolumn{4}{|c|}{ Fjordings } \\
\hline étalon & $x$ & jument & produits \\
\hline FI & & $\begin{array}{ll}\mathrm{G} & (1) \\
\mathrm{GI} & (7) \\
\mathrm{I} & (2)\end{array}$ & $\begin{array}{l}\text { FG (2), GI (2) } \\
\text { FG (4), FI (1), GI (1), I (3) } \\
\text { FI (2), F (1)* }\end{array}$ \\
\hline I & $x$ & $\begin{array}{l}\mathrm{G}(2) \\
\mathrm{GI} \quad(1) \\
\mathrm{I} \quad(2)\end{array}$ & $\begin{array}{ll}\text { GI } & (2) \\
\text { GI } & (1) \\
\mathrm{I} & (2)\end{array}$ \\
\hline
\end{tabular}

* Filiation incompatible ou génotype $\mathrm{F} / \mathrm{O}$.

\begin{tabular}{|c|c|c|c|}
\hline \multicolumn{4}{|c|}{ Huculs } \\
\hline étalon & $x$ & jument & produits \\
\hline FS & & $\begin{array}{l}\text { FS (1) } \\
\text { F (2) } \\
\text { FI (3) } \\
\text { IS (1) } \\
\text { I (4) }\end{array}$ & $\begin{array}{l}\text { FS (1), IS (1)** } \\
\text { F (1), FS (3) } \\
\text { F (1), FI (2) } \\
\text { FS (1), FI (1) } \\
\text { FI (4), IS (4) }\end{array}$ \\
\hline I & & $\begin{array}{l}\text { F (1) } \\
\text { FI (1) } \\
\text { IS (1) }\end{array}$ & $\begin{array}{l}\text { FI (8) } \\
\text { FI (1) } \\
\text { IS (1) }\end{array}$ \\
\hline
\end{tabular}


une jument de phénotype I, l'un des produits est FI et l'autre $\mathrm{F}$. Ce dernier résultat peut être dû soit à une généalogie erronée soit à la présence chez la jument de 1'allèle récessif Es ${ }^{\circ}$ : le génotype du produit serait alors $\mathrm{F} / \mathrm{O}$.

Pour les Huculs, l'étude de la transmission, effectuée sur la descendance de deux étalons, a conduit à une exclusion : FS $\times$ FS donne IS (tabl. 7 , fig. I). Dans un autre cas $F S \times F$ donnant $F$, on se trouve peut être en présence de l'allèle silencieux.
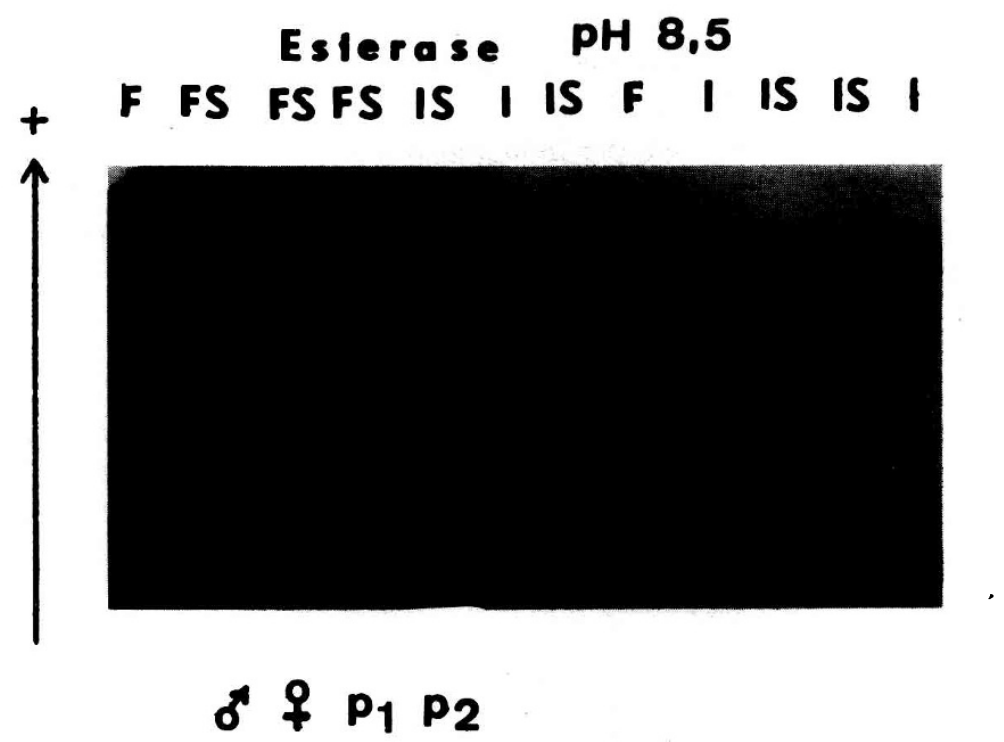

Frg. r. - Phénotypes d'estérase à pH 8,5

Cas de fausse filiation : le produit $n^{\circ}$ I57 étant IS ne peut descendre de l'étalon $n^{\circ}$ I59 qui est FS. La jument (15I) et l'autre produit (13I) sont également FS

La fréquence de Es $S^{F}$ est très élevée : 37 p. roo ; parmi les résultats obtenus dans notre laboratoire, la valeur la plus élevée a été trouvée pour les chevaux de trait Bretons : 29 p. roo et Ardennais : 22 p. Ioo (KAMInski, I974). Pour comparer ce résultat à ceux d'autres auteurs il faut tenir compte de l'absence de Es ${ }^{G}$ chez les Huculs et de sa présence possible dans d'autres races, étudiées à $\mathrm{pH} 8,5$. On constate que le seul taux supérieur à $37 \mathrm{p}$. Ioo est celui trouvé chez les Percherons en Afrique du Sud (OSTERHoff et al., r967) : 45 p. I0o ; or, les Percherons possèdent une fréquence élevée

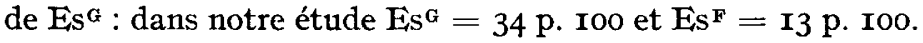

Ainsi les $H u c u l s$ apparaissent comme la race la plus riche en Es E étudiée jusqu'ici. Remarquons à ce propos que la population étudiée comprenait I2 juments demisœurs, toutes possédant l'allèle $F$ à simple ou double dose. Ces juments ont donné, avec 3 étalons, dont I possédait $F, 2$ I produits. De ces 2 I produits seulement 3 ne possédaient pas $F$, et l'un d'eux est un cas d'exclusion (voir plus haut).

Le taux de $\mathrm{Es}$ s : I5 p. Ioo est également fort, comparé aux autres races.

La proportion globale d'hétérozygotes pour l'estérase est extrêmement élevée chez les Huculs (surtout pour une population consanguine) : $7 \mathrm{I}$ p. 100.

Parmi les enzymes intra-érythrocytaires, la seule différence entre les Fjordings et les Huculs a été observée au niveau de la phosphohexose isomérase : chez les 
Fjordings on a trouvé $2 \mathrm{~F}, 37 \mathrm{FI}, 23$ I et I IS ; chez les Huculs, I9 FI et 62 I. La fréquence de $\mathrm{F}$ est donc bien supérieure chez les Fjordings. Les autres systèmes étudiés se sont montrés pratiquement monomorphes : phosphoglucomutase, 6-phosphogluconate déshydrogénase et phosphatase acide.

\section{DISCUSSION ET CONCLUSIONS}

Malgré le nombre peu élevé d'échantillons examinés et la consanguinité des populations étudiées, la répartition de la plupart des allèles paraît bien caractéristique de chacune des races en question. Cependant, la comparaison de nos résultats avec les données obtenues pour d'autres races est parfois difficile à établir ; ainsi dans le cadre du système $D$ des groupes sanguins, les chevaux de trait n'ont pas été suffisamment étudiés et le nombre exact et la nature de leurs allèles restent encore à préciser.

On note dans les deux races, mais surtout chez les Fjordings, une tendance à l'hétérogénéité restreinte : pour certains systèmes génétiques, tels le système $\mathrm{A}$ des groupes sanguins ou les transferrines, le nombre total d'allèles ou de phénotypes observé est moins élevé que dans d'autres races. Ceci n'est pourtant pas dû à un isolement prolongé, étant donné des apports extérieurs, ni à l'effet " du fondateur " car les deux races se sont formées sur place, lentement et non à partir d'un groupe restreint.

A côté de cette restriction du nombre d'allèles, on remarque une proportion importante d'hétérozygotes, pour les deux systèmes sériques multi-allèliques : transferrine et estérase.Ceci peut être dû seulement au hasard de l'échantillonnage et nous avons indiqué que les populations testées n'étaient pas panmictiques. Cependant il pourrait en outre s'agir de l'avantage des hétérozygotes.

Au sujet d'allèles manquants, soulignons l'absence de $\mathrm{Tf}^{\mathrm{M}}$, qui paraît ainsi circonscrit aux poneys Shetland et Islandais.

Dans l'ensemble, les Fjordings et les Huculs se rapprochent plutôt des chevaux de trait, ce qui peut paraître logique vu leur origine, mais il n'y a pas de similitudes frappantes. De plus, il n'y a pas de parenté évidente entre ces deux races et d'autres Poneys.

Entre les Fjordings et les Huculs les différences les plus nettes sont le fait de la répartition de quelques allèles de groupes sanguins de systèmes $\mathrm{A}$ et $\mathrm{D}$, d'estérase et dans une moindre mesure de transferrine.

On peut penser que, malgré les croisements divers, dus à l'homme, qu'ils ont subis, la physionomie phénotypique propre de chaque race résulte surtout de sa lointaine origine et des influences de l'environnement dans lequel elle s'est fixée.

Ręu pour publication en mai 1975.

\section{REMERCIEMENTS}

Ce travail a bénéficié du support financier de la Société d'Encouragement pour l'Amélioration des races de chevaux en France (Directeur J. Romanet).

Nous remercions M. F. Grosclaude pour les remarques et la discussion du manuscrit. 


\section{SUMMARY}

\section{BLOOD GENETIC MARKERS IN TWO BREEDS OF PONEYS FROM POLAND}

3 I erythrocyte antigens (blood group factors) belonging to 7 genetic systems and 4 polymorphic proteins and enzymes of serum and haemolysate, were investigated in 63 Fjordings and 8I Huculs, both bred in Poland.

Marked differences between the two breeds were demonstrated concerning the repartition of factors, phenotypes or alleles from several genetic systems.

The comparison with other horse breeds showed similarities with draught horses rather than with light horses.

\section{RÉFÉRENCES BIBLIOGRAPHIQUES}

Bengtsson S., Gahne B., Rendel J., 1968. Genetic Studies on Transferrins Albumins, Prealbumins and Esterases in Swedish Horses. Acta Agr. Scand., 18, 60-64.

BRAEND M., 1964. Serum types of Norvegian horses. Nord. vet. Med., 16, 363-373.

Braend M., 1973. Genetic Variation in Equine Blood Proteins. Proc. 3rd int. Conf. Equine Infections Diseases, Paris, 1972, Karger ed., 394-406.

Buis R. C., I973. Horse Blood Typing in the Netherlands. Rapport présenté à la Réunion de C. O. P. A., Paris.

Dobrev D., Ermencova L., Karaivanov R., Tsankov Ts., 1972. Determination of blood groups and transferrins in three breeds of horses in Bulgaria. Proc. XIIth Europ. Conf. Anim. Blood Groups Biochem. Polymorph. (Budapest, 1970), p. 537-540. W. Junk, the Hague.

Hesselholt M., I966. A study of blood groups and serum types of the icelandic horse. Proc. Xth Conf. Eur. Soc. Anim. Blood Grps. Res., Paris, I. N. R. A., 325-33I.

Kaminski M., I975. Polymorphisme de transferrine et d'estérase chez le Cheval, Comm. présentée à la Table Ronde sur les Polymorphismes Biochimiques chez les Mammifères, Orléans I973; éd. C. N. R. S. (sous presse).

Kaminski M., Bouguet Y., Van De Weghe A., Podliachouk L., r974. Ontogenèse des marqueurs génétiques sanguins chez le Cheval. Ann. Génêt. Sêl. Anim., 6 (2), I95-210.

Kaminski M., Van De Weghe A., Bouquet Y., Podliachouk L., r975. Marqueurs génétiques sanguins chez les chevaux de trait (en préparation).

LARSEN B., I97I. Blood groups and polymorphic proteins in cattle and swine. Ann. Génét. Sél. anim., 3, 59-70.

OSterhoff D. R., WARD-Cox F. S., 1967. A preliminary horse breed comparison with regard to haemoglobin and serum type polymorphism. Proc. S. Afr. Soc. Anim. Prod., 218-223.

OsterhofF D. R., Schmid D. O., WARD-Cox F. S., I970. Blood group and serum type studies in Basuto Ponies. Proc. XIth Europ. Conf. Anim. Blood Groups Biochem. Polymorph. (Warsaw, I968), p. 452-457. W. Junk, The Hague.

Podlachouk L., 1957. Les antigènes des groupes sanguins des Equidés et leur transmission héréditaire. Thèse Doct. ès-Sci., Paris.

Podliachouk L., Kaczmarek A., Zwolinski J., I962. Les groupes sanguins des chevaux des 6 races de Pologne. Ann. Inst. Pasteur, 103, 943-949.

Podliachouk L., Kaminski M., 1972. Studies on blood groups, esterases and transferrins in light and draught horses. Comm. présentée à la XIII' Conf. ISABR, Vienne. Anim. Blood Grps. Biochem. Genet., 8, suppl. I, 46-47.

Podliachouk L., Kaminski M., Bouguet Y., Zwolinski J., Van de Weghe A., Siudzinski S., 1974. Blood genetic markers in race horses. Comm. présentée à la XIVe Conf. ISABR, Davis.

Podliachouk L., Van de Weghe A., Bovquet Y., Kaminski M., Zwolinski J., 1975. Marqueurs génétiques sanguins chez les clıevaux de courses. Ann. Génét. Sel. anim. (sous presse).

SandberG K., 1973. The D blood group system of the horse Anim. Blood Grps biochem. Genet., 4, 193-205.

SANDBERG K., I974. Blood Typing of Horses : current status and application to identification problems. Proc. I Congrès Mondial de Génétique appliquée à l'Élevage. Madrid, 1974, t. I, Sessions Plénières, 253265. 
Schleger W., 1974. Biochemische Genepolymorphismen im Oesterreichischen Lippizanerpferd. Proc. I Congrès Mondial de Génétique appliquée à l'élevage. Madrid, 1974, t. 3, Symposia, 24I-247.

Stormont C., Suzuki Y., 1964. Genetic systems of blood groups in horses. Genetics, 50, 915-929.

Tomaszewska-Guszkiewicz K., Zurkowski M., r97o. Transferrin and Albumin Polymprphism in the polish "Tarpan " breed. Proc. XIth Europ. Conf. Anim. Blood Groups Biochem. Polymorph. (Warsaw, r968) p. 473-475. W. Junk, The Hague. 